\title{
Retroperitoneal fibrosis with normal intravenous urogram
}

\author{
F.M. Creagh ${ }^{1}$, T. Stone ${ }^{2}$, T.P. Stephenson ${ }^{2}$ and J.H. Lazarus ${ }^{1}$ \\ Departments of ${ }^{\prime}$ Medicine $\&^{2}$ Urology, Llandough Hospital, Penarth, S. Glamorgan and Cardiff Royal Infirmary, \\ Newport Road, Cardiff, Wales.
}

\begin{abstract}
Summary: A 58 year old male presented with a two week history of low back pain and malaise. The intravenous urogram (IVU) at presentation was normal but within three months he had developed renal failure with bilateral ureteric obstruction on repeat IVU. Primary retroperitoneal fibrosis was confirmed at operation.

This case demonstrates that retroperitoneal fibrosis may progress rapidly to renal failure within a few months of the first symptoms. In addition, the IVU may be normal in the early stages of the illness.
\end{abstract}

\section{Introduction}

Retroperitoneal fibrosis is an insidious disease most frequently occurring in men in their sixth decade. Delay in diagnosis is common and has been attributed to the nonspecific nature of the presenting symptoms malaise, back pain, and weight loss. In the majority of cases however, the diagnosis is suggested by an abnormal intravenous urogram (IVU). We report a case in whom an IVU early in the course of the illness was normal and in whom rapid onset of renal failure associated with bilateral ureteric obstruction developed.

\section{Case report}

A 58 year old male physiotherapist presented as a surgical emergency in November 1981 with a two week history of severe low back pain associated with malaise, anorexia and seven pounds weight loss. There was no relevant past medical or drug history. There were no physical findings, and full blood count, urea and electrolytes, and serum amylase were all normal. ESR was elevated at $45 \mathrm{~mm} / \mathrm{h}$. An intravenous pyelogram one week later was reported as normal (Figure 1). During the subsequent two months he remained severely disabled by back pain requiring frequent administration of pethidine or dihydrocodeine. Lumbar spine radiographs, bone scan and barium enema were all normal. By January

F.M. Creagh, M.B., M.R.C.P.; T. Stone, F.R.C.S.; T.P. Stephenson, M.S., F.R.C.S.; J.H. Lazarus, M.D., F.R.C.P. Correspondence: J.H. Lazarus, Department of Medicine, Llandough Hospital, Penarth, S. Glam.

Accepted: 10 January 1984
1982, he had lost 2 stone in weight but the back pain had begun to subside.

In February 1982, he was admitted as a medical emergency with a three week history of severe early morning headaches, shortness of breath and ankle

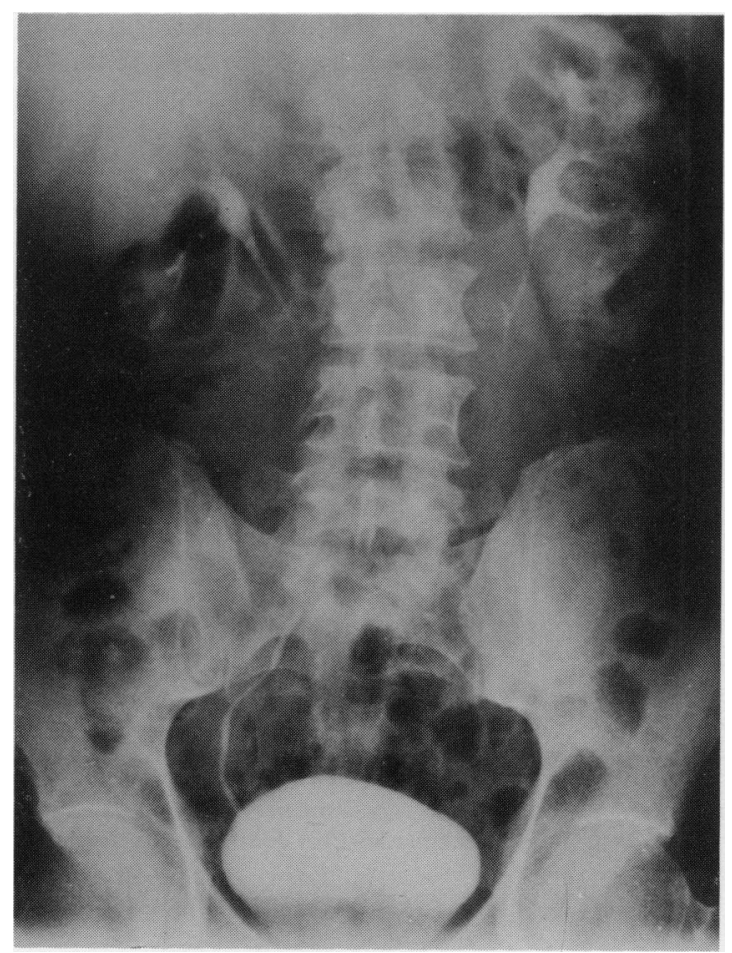

Figure 1 Normal IVU - November 1981.

(C) The Fellowship of Postgraduate Medicine, 1985 
oedema. On examination the blood pressure was $220 /$ $120 \mathrm{~mm} \mathrm{Hg}$ with no evidence of retinopathy. He was clinically anaemic and in congestive cardiac failure. There were no signs on abdominal examination. Investigations confirmed anaemia (haemoglobin $9.2 \mathrm{~g} / \mathrm{l})$ elevated ESR at $53 \mathrm{~mm} / \mathrm{h}$; blood urea was $20 \mathrm{mmol} / 1$ and creatinine $0.59 \mathrm{mmol} / 1$. The mid-stream urine (MSU) was normal. Chest X-ray revealed cardiomegaly with bilateral pleural effusions and interstitial oedema. Repeat IVU demonstrated bilaterally enlarged kidneys with hydronephrosis, hydro-ureter and a normal sized bladder (Figure 2). Retrograde pyelography showed the typical appearances of retroperitoneal fibrosis and was followed by urgent laparotomy. At operation a typical retroperitoneal plaque was identified overlying the aorta and invading the ureters which were displaced medially. The ureters were freed and wrapped in omentum. Ten days postoperatively urea and creatinine levels had fallen to normal and the blood pressure was controlled on a small dose of hydralazine. Two months later the patient was entirely well on no medication, and ESR was normal.

\section{Discussion}

This case illustrates that the IVU may be normal in the early stages of retroperitoneal fibrosis. A normal IVU has been reported in $2.5-7 \%$ of cases at presentation (Lepor \& Walsh, 1979). This finding is compatible with the pathological features of the disease: the early phase is characterized by an inflammatory reaction and it is only in the later stages, as fibrosis develops, that ureteric obstruction occurs (Cullen, 1981). The ESR is thought to reflect the activity of the inflammatory reaction (Abercrombie \& Vinnicombe, 1980) and it is noteworthy in this case that the ESR was elevated from the outset.

Retroperitoneal fibrosis is frequently thought to be a slowly progressive disease (Mitchinson, 1982). However, in this case renal failure developed within three months of the first symptoms. Rapid radiological progression from normal to severe obs-

\section{References}

ABERCROMBIE, G.R. \& VINNICOMBE, J. (1980). Retroperitoneal fibrosis. Practical problems in management. British Journal of Urology, 52, 443.

CULLEN, T.H. (1981). Retroperitoneal fibrosis - an association with hyperuricaemia. British Journal of Surgery, 68, 199.

LEPOR, H. \& WALSH, P.C. (1979). Idiopathic retroperitoneal fibrosis. British Journal of Urology, 122, 1.

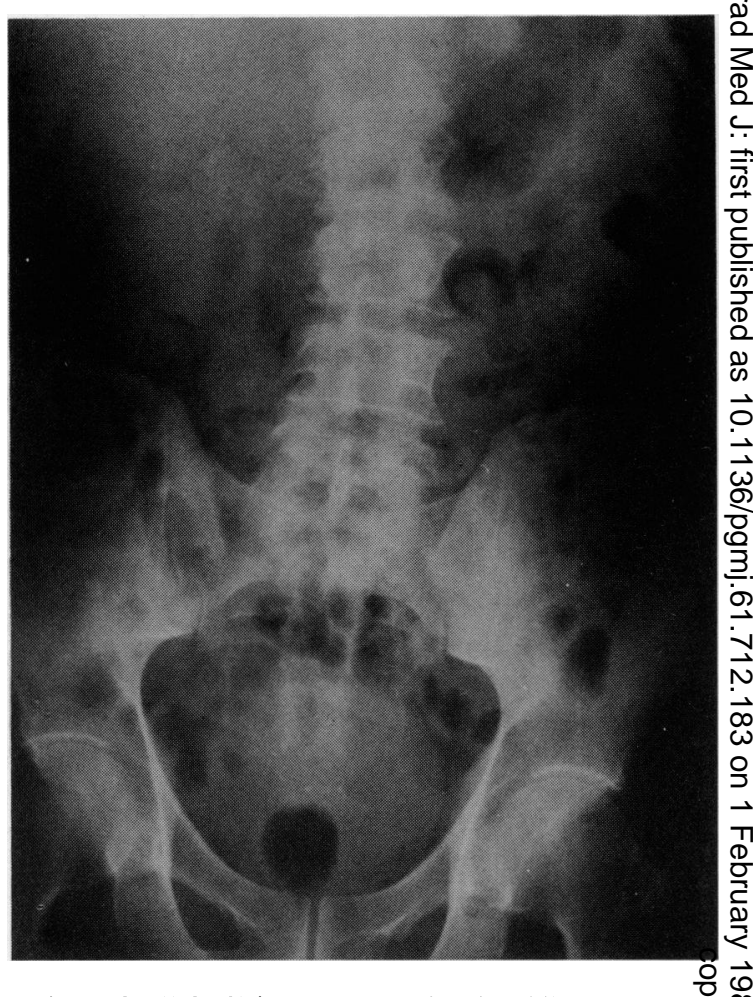

Figure 2 IVU February 1982 showing bilateral renals o enlargement with hydronephrosis, hydro-ureter. Bladder size is normal.

truction within two to five months has also been reported (Utz \& Henry, 1966).

This case serves as a reminder that retroperitoneal fibrosis may be a rapidly progressive disease and that a normal IVU does not rule out the diagnosis. Elevation of the ESR is a hallmark of the disease. Retroperitoneal fibrosis should, therefore, be considered in the differential diagnosis of any patient presenting with $\frac{3}{3}$ back pain and an elevated ESR, in spite of a normal IVU.

MITCHINSON, M.J. (1982). Retroperitoneal fibrosis N associated with metoprolol. British Medical Journal, 284, 347.

UTZ, D.C. \& HENRY, J.D. (1966). Retroperitoneal fibrosis. స్ట Medical Clinics of North America, 50, 1091. 